\title{
De la técnica a la tecnología: construcción de forjados de cerámica abovedada mediante sistemas industrializados
}

\section{The art of the technology: construction of structures by domed ceramic with industrial systems}

$\underline{\text { J. Trias de Bes }}^{(*)}, \underline{\text { P. Casariego }}{ }^{(*)}$

RESUMEN

La conciliación entre la construcción tradicional y las nuevas tecnologías motivan el desarrollo de un nuevo entrevigado abovedado con revoltón de ladrillo mediante una tecnología de prefabricación de hormigón armado en el que se incorpora en el proceso constructivo un tejido cerámico. Se muestra el ejemplo de una vivienda prototipo construida con este sistema.

El artículo presenta dos reflexiones principales: a) Exponer la influencia de la imaginería de las técnicas tradicionales como la motivación y la activación de los procesos tecnológicos de la construcción. b) Confrontar Prefabricación u Ordenamiento vs. Oficio como procesos constructivos pertenecientes a la tecnología y a la técnica, respectivamente. En este sentido, el artículo concluye poniendo el énfasis que el humanismo media entre ambas dentro del proceso proyectual arquitectónico.

Palabras clave: técnica; tecnología; procesos tecnológicos; Ordenamiento vs. Oficio, humanismo de la tecnología.

\section{ABSTRACT}

The reconciliation between traditional construction and new technologies encourage the development of a new catalonian vaulted through prefabrication technology of reinforced concrete in which it is incorporated in the construction process a ceramic fabric. The example of a prototype house with this system is shown.

The article presents two fundamental considerations: a) Expose the influence of the imagery of traditional techniques as motivation and activation of technological construction processes, and b) confront System Vs. Skill as a constructive belonging to the Technical and Technology, respectively. In this sense, the article concludes by highlighting that humanism is involved between both as a determinant factor in the architectural design process.

Keywords: technique; technology; technological process; System Vs. Skill; humanism technology.

(*) Universitat Internacional de Catalunya (UIC). School of Architecture Dept. Architectural Construction, Spain. Personas de contacto/Corresponding authors: jtrias@uic.es; juan@tdb-arquitectura.com; pcasariego@uic.es

Cómo citar este artículo/Citation: Trias de Bes, J., Casariego, P. (2016). De la técnica a la tecnología: construcción de forjados de cerámica abovedada mediante sistemas industrializados. Informes de la Construcción, 68(544): e169, doi: http://dx.doi.org/10.3989/ ic.15.168.m15.

Copyright: (C) 2016 CSIC. Licencia / License: Salvo indicación contraria, todos los contenidos de la edición electrónica de Informes de la Construcción se distribuyen bajo una licencia de uso y distribución Creative Commons Attribution License (CC BY) Spain 3.o. 


\section{INTRODUCCIÓN. ANTECEDENTES}

En este artículo se presenta el desarrollo original de un sistema prefabricado de bóveda catalana. Teniendo en cuenta el gran arraigo que esta bóveda tiene en la cultura de la geografía catalana, y en gran parte de Latinoamérica, resulta pertinente exponer sus orígenes y evolución a fin de contextualizar la experimentación llevada a cabo.

A grandes rasgos podríamos decir que hay tres tipos de bóvedas realizadas con cerámica: 1) la bóveda catalana original; 2) la cerámica armada de Eladio Dieste, y 3 ) las experiencias con bóvedas prefabricadas de ladrillo llevadas a cabo en los años 60 y 70 por arquitectos como Eduardo Sacriste o GonzálezLobo.

\subsection{La tradición}

La bóveda catalana o bóveda tabicada consiste en una primera hoja, denominada «sencillo», en la que las rasillas se disponen de tabla y se reciben con pasta de yeso, que tiene la capacidad de fraguar y adquirir su resistencia rápidamente.

Esta característica de la pasta de yeso permite a esta primera hoja aguantarse en vilo. Esto es, no requiere encofrado ni cimbra. Así pues, es factible trazar la geometría «al aire», aunque en ciertas ocasiones se emplean cimbras deslizantes para asegurar el correcto trazado.

Esta primera hoja se construye por tramos, donde cada tramo no debe exceder los $60 \mathrm{~cm}$; esto es, no debe superar la longitud aproximada del brazo de un operario, ya que sobre ella se ha de colocar la segunda hoja, denominada «doblado». El doblado se debe colocar de tal forma que haya discontinuidad respecto a las juntas del sencillo, por lo que es muy habitual que esta segunda capa se disponga a $45^{\circ}$ respecto a la primera.

Estas dos capas aparejadas se pueden entender como un tabique. De ahí que a la bóveda catalana se le denomine también «bóveda tabicada», y que algunos autores, como Bassegoda Musté (1), definan la bóveda catalana como aquella «formada por ladrillos puestos de tabla, unos a continuación de otros en toda su vuelta, cintra o curvatura, de modo que viene a ser toda ella como un tabique».

La bóveda catalana tiene un origen muy antiguo en Cataluña y está fuertemente arraigado en su cultura social. La arquitectura de Gaudí, los edificios modernistas de Barcelona, el Dispensario Antituberculoso de Sert (2) y muchos de los edificios del Ensanche barcelonés, por poner algunos ejemplos, están construidos con esta técnica.

El ingenio de este sistema constructivo, su economía y accesibilidad (la arcilla se encuentra en casi todo el planeta), ha provocado su expansión a otros países, como EE. UU., Cuba y gran parte de Latinoamérica.

\subsection{La evolución. La experiencia latinoamericana}

Aunque no se sabe con certeza cómo se produjo la expansión de la bóveda catalana a otros países, es muy factible que el salto se haya producido de la mano de emigrantes; por ejemplo, Guastavino (3) (4) que en 1881 emigra a EE. UU. con su hijo y cuatro años más tarde, en 1885 , patenta el sistema constructivo la bóveda catalana o bóveda tabicada de ladrillo plano.

En el caso de Cuba podemos encontrar a figuras como el caso del maestro albañil catalán Gumersindo, que llegado a este país tras la victoria de la Revolución en 1959, su primera labor consiste en formar albañiles para aprender la técnica de construcción de la bóveda catalana. Con ella se construyen las Escuelas Nacionales de Arte de la Habana (5). Cabe mencionar que su oficio lo aprendió de su padre, quien a su vez había trabajado con Gaudí.

Una figura muy relevante en lo que a la evolución de la bóveda catalana se refiere es la de Antoni Bonet, que funda el GATECPAC junto con J. L. Sert, ingresa en el estudio de Le Corbusier en 1936 cuando estalla la Guerra Civil, y emigra a Buenos Aires en 1939 tras la finalización de la misma debido a sus ideas políticas.

Para la construcción de la casa Berlinghieri (1947), en Punta Ballena (Uruguay), Bonet contacta con Eladio Dieste para que calcule la estructura, de donde surge la concepción de la cerámica armada. Tal y como expone García. J. (6), «es un hecho académicamente aceptado que las bóvedas de Bonet y Dieste en la Berlinghieri fueron las primeras en combinar cerámica y armaduras de acero».

Así pues, la cerámica armada se puede considerar una evolución de la utilización de la bóveda cerámica tradicional.

En el caso de la casa Berlinghieri el sistema aún es un poco más evolucionado, dado que se construye una cámara de aire con acabado cerámico. De hecho, tal y como expone Prozorovich (7), la evolución de la bóveda catalana en Bonet se puede observar a través de cuatro casas: Martínez (1942), casa Berlinghieri (1947), pabellón Berlinghieri (1951) y la Ricarda (1953), donde la "sección había evolucionado desde una bóveda simple de hormigón a una doble bóveda compuesta por una hoja de hormigón aligerado con ladrillo y una segunda bóveda no estructural que cierra una cámara aislante y resuelve la evacuación de aguas", (Figura 1, izquierda). En este caso, las bóvedas cubren luces medias sin pilares intermedios, por lo que hay una clara diferencia respecto a la bóveda catalana dispuesta en los entrevigados de forjados.

Le Corbusier también empleará una versión de la bóveda catalana en la casa Jaoul (Figura 1, derecha) y Sarabhai, ambas de 1955, posiblemente influido por su relación con Bonet tal y como observa García, J. (8). Las bóvedas de ambas casas construidas con ladrillos dispuestos en tabla alcanzan ciertas dimensiones. En los casos en que los empujes no son contrarrestados por las bóvedas adyacentes se disponen tirantes.

\subsection{Los sistemas prefabricados}

La modernidad desplegada por las obras abovedadas de Bonet, Dieste y Le Corbusier, entre otros, ejerce gran influencia en los años 60 y 70 en arquitectos como el argentino Eduardo Sacriste.

Autor del libro Viviendas con bóveda (9), Sacriste experimenta con todo tipo de bóvedas, desde la tabicada tradicional, pasando por la cerámica armada en la casa Larrán y casa 

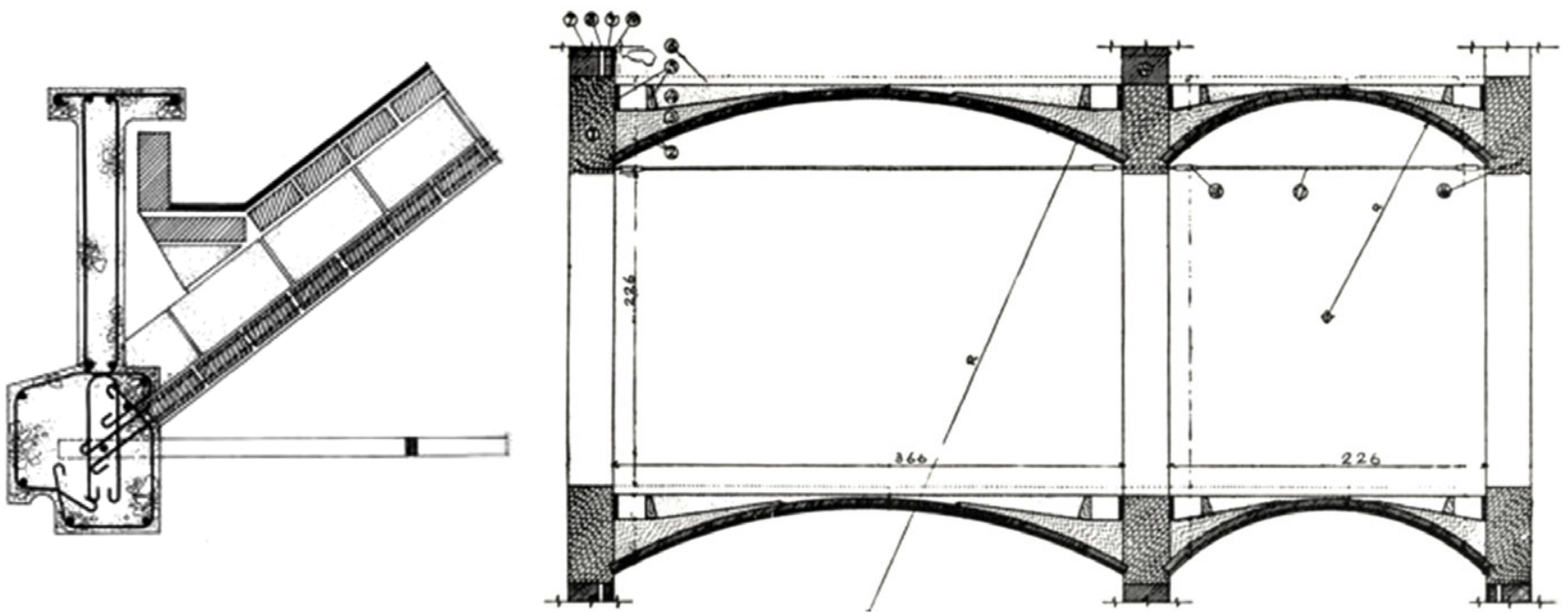

Figura 1. Detalle constructivo casa Berlinghieri (izquierda); casa Jaoul (derecha).
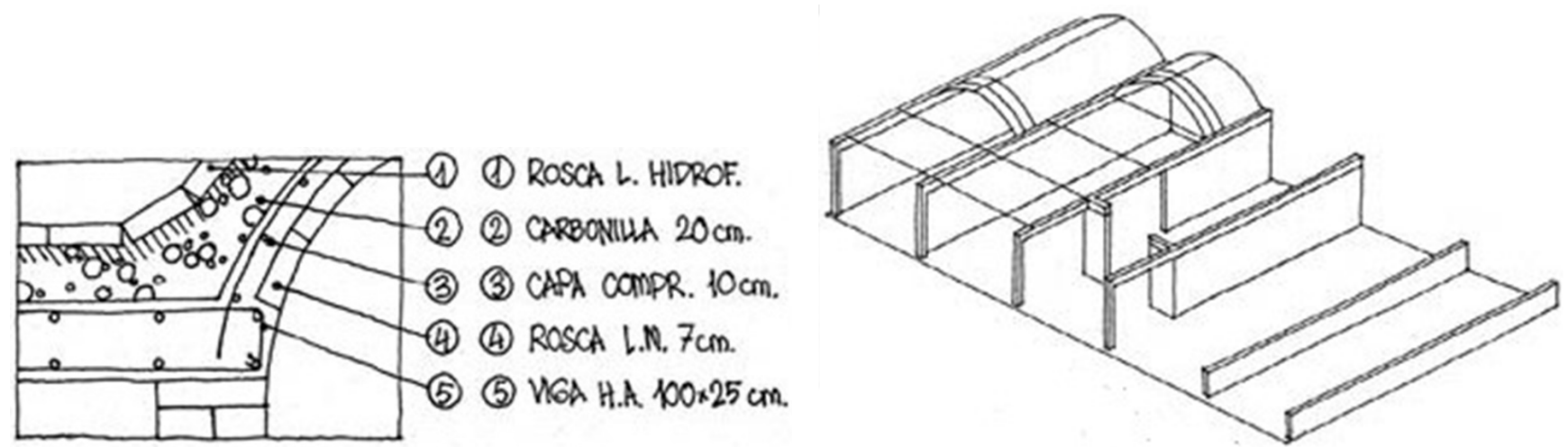

Figura 2. Detalle constructivo casa Gayraud (izquierda); casa Carrieri (derecha).

Gayraud (Figura 2, izquierda), y acabando con sistemas prefabricados en las casas Carrieri (Figura 2, derecha).

Tal y como el propio arquitecto expone en Viviendas con bóveda (9), acerca de la casa Carrieri:

«Para la construcción de las bóvedas se utilizaron "costillas" armadas y prefabricadas in situ sobre cuatro moldes de curvas ajustadas según plantillas dobles. Sobre las superficies de los moldes marcados y engrasados previamente se iban disponiendo mitades de ladrillos huecos. Luego de construidas y curadas, estas unidades autorresistentes se apoyaban directamente en los muros longitudinales, sin utilizar cimbras y conectando los hierros de las viguetas con las armaduras invertidas de encadenado superior».

También destaca el trabajo del mejicano González-Lobo, que incluso desarrolla patentes (GGL-1, GGL-2), que emplea en muchas construcciones (8) (10) (11). Sobre un molde curvo a nivel de suelo se disponen ladrillos pareados. En la junta horizontal se dispone una varilla de acero, mientras que en las transversales se arman tres alambres recocidos por junta y se vierte un mortero simple. La clave de la bóveda está conformada por un zuncho de acero al que se «atan» las piezas prefabricadas. En la parte superior se vierte una capa de compresión armada con acero (Figura 3).
En cuanto a tamaño y procedimiento cabe destacar también la obra del uruguayo Mario Kalemkerian, que plantea elementos prefabricados de gran tamaño, para cuya construcción y transporte despliega un gran ingenio. Es el caso, por ejemplo, de la cúpula de 8 metros de diámetro construida para el complejo del Arsenal de la Armada en Montevideo (11).

Cabe mencionar aquí el programa internacional CYTED (Ciencia y Tecnología para el Desarrollo) [12], creado en 1984 por acuerdo entre los gobiernos de 21 países iberoamericanos, que promueve la mejora de calidad de vida. Dentro del mismo se acoge el programa $10 \times 10$, que consiste en la creación de 100 viviendas que recojan las tecnologías de diferentes países. Las más tradicionales, racionalizadas o innovadoras, y que supongan una aportación para resolver las viviendas del país donde se construye y no generen dependencia tecnológica.

Dentro de las soluciones aportadas se encuentran diferentes soluciones prefabricadas, como el techo BATEA, la semibóveda de cerámica armada CEVE, el sistema LAM, o el muro BENO, (Figura 4).

Algunas de ellas recuerdan al sistema de prefabricación de González Lobo, mientras que entroncan con el proceso de prefabricación empleado en el sistema constructivo presentado en este artículo. 

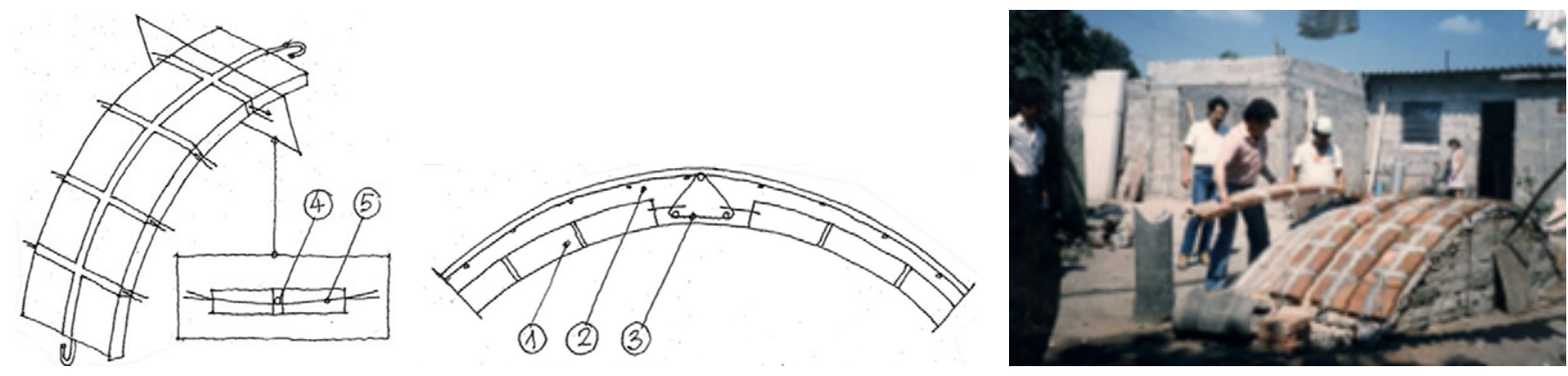

Figura 3. Prefabricados González-Lobo.
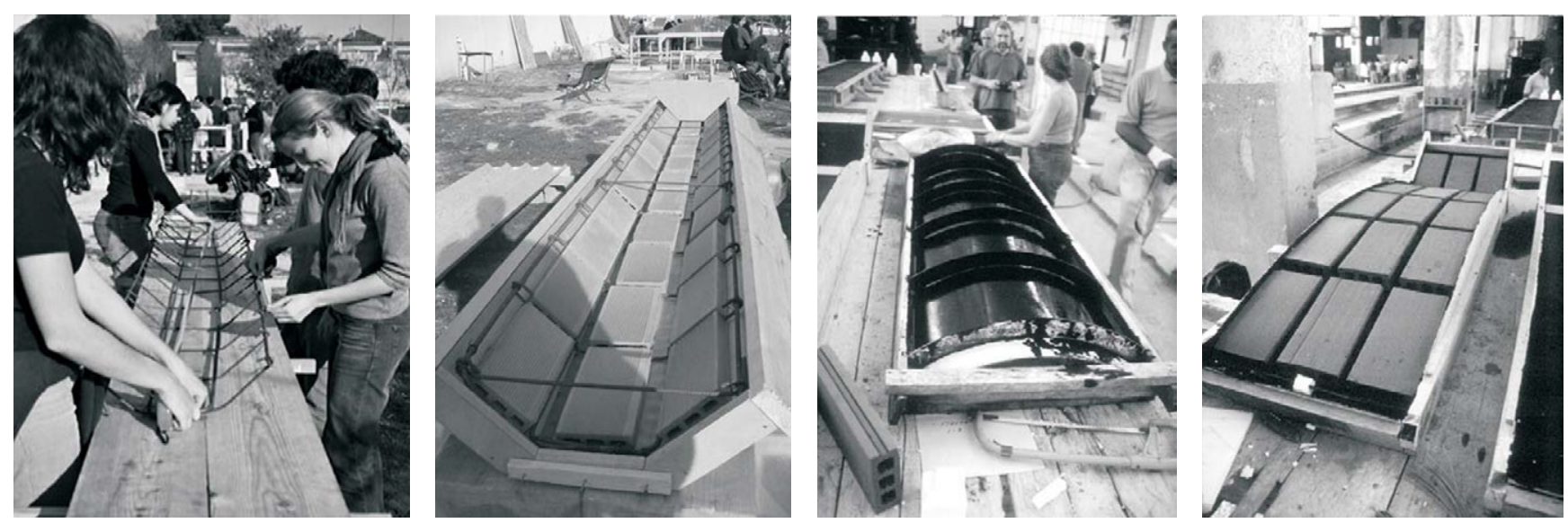

Figura 4. De izquierda a derecha: armado techo BATEA; techo BATEA antes de hormigonar; sistema LAM; semibóveda CEVE.
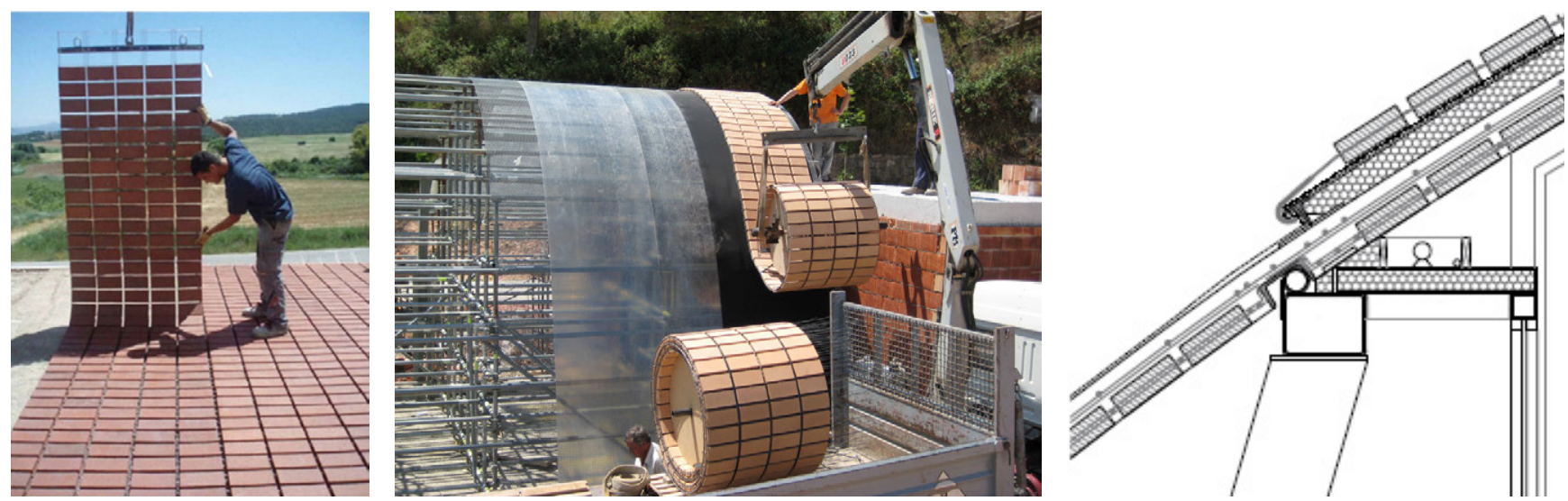

Figura 5. Sistema Flexbrick (izquierda); construcción bóveda de casa Mingo (centro); detalle cubierta casa Mingo (derecha).

Por último, cabe destacar en España la solución prefabricada Flexbrick (13), del arquitecto V. Sarrablo, cuyo sistema está basado en el concepto de láminas cerámicas flexibles que pueden adaptarse a cualquier forma. Básicamente, consiste en una malla de acero trenzado que sujeta las piezas de arcilla cocida y que alcanza longitudes de hasta $20 \mathrm{~m}$ (Figura 6, izquierda). El sistema evita la tradicional colocación a mano de cada una de las piezas, lo que permite ganar tiempo de ejecución. Esta solución admite diferentes diámetros de acero ente las juntas, por lo que la malla puede emplearse como un simple revestimiento, o como cerámica armada para la construcción de arcos y bóvedas. Un ejemplo de ello sería la casa Mingo (14) (15) cuya cubierta se resuelve con dos tipos de malla. Una exterior que actúa como revestimiento, y una interior que actúa como cerámica armada y resuelve la bóveda. Entre ambas capas se incorporan los elementos constructivos necesarios para solucionar los problemas de aislamiento (Figura 5).

\section{LA CONCEPCIÓN DEL SISTEMA PREFABRICADO}

\subsection{El dilema}

El dilema inicial se produce en el propio paso de un sistema constructivo tradicional a un sistema prefabricado.

Inicialmente, la idea es desarrollar un sistema prefabricado tipo «bovedilla catalana» de escasos centímetros de grosor que se aloja entre viguetas y trabaja como un arco. Sin embargo, este sistema constructivo se contradice directamente con el propio sentido de la prefabricación, ya que la colocación de 
viguetas en obra implica un tiempo de ejecución que resulta incompatible con un sistema prefabricado, en términos de eficacia.

Esta circunstancia conduce a incluir las viguetas dentro del propio sistema prefabricado. Teniendo en cuenta que finalmente se ha de formalizar un relleno, una capa de compresión y asegurar el monolitismo del forjado, se opta por emplear hormigón; reduciendo el número de materiales a utilizar. Así pues, la idea inicial evoluciona hacia emplear la cerámica y el hormigón conjuntamente; emulando a pequeña escala la solución constructiva de Le Corbusier para la casa Jaoul.

Como siguiente paso se busca la manera de sistematizar la construcción del revoltón cerámico, por lo que se acude a la patente Flexbrick. El sistema posee las cualidades requeridas buscadas: se adapta a la geometría de la bóveda, permite realizar piezas de grandes longitudes, evita la colocación a mano de cada una de las piezas y puede trabajar como cerámica armada.

La solución adoptada consiste en un sistema industrializado, considerando toda la sección de hormigón como una gran viga que trabaja unidireccionalmente. Así, se optimiza el acero, evitando las dobles viguetas. Al mismo tiempo se facilita la ejecución al emplear un solo material y no requerir encofrados complejos.

Ello implica que la solución final pasa por ser una losa prefabricada de hormigón de sección abovedada con acabado cerámico.

\subsection{Cálculo, dimensionado y armado}

El armado de la losa del forjado para el hormigonado in situ se realizó según el esquema que se muestra en la Figura 6.

El armado se calcula para las dos fases a las que estará solicitada la «viga abovedada»: 1) La fase de acopio y transporte, y 2) la fase de trabajo como forjado, propiamente dicho. En la primera fase las losas trabajan biapoyadas soportadas por el armado que recorre perimetralmente la longitud de la losa. Este armado está conformado por redondos longitudinales de Ø8 y redondos transversales de $\varnothing_{5}$ separados cada $15 \mathrm{~cm}$. El canto máximo de la losa es de $20 \mathrm{~cm}$ y el mínimo de $7,4 \mathrm{~cm}$. Con ello se asegura que no se produzcan fisuras en el hormigón.

En la fase de obra in situ se vierte el hormigón para conformar la capa de compresión y asegurar el monolitismo del forjado. Una vez fragua el hormigón, el forjado entra en carga. Las armaduras de conexión están ejecutadas con redondos de Ø10 y $\emptyset_{12}$, los negativos de Ø10 están ejecutados in situ.
La losa final tiene $26 \mathrm{~cm}$ de canto máximo y $14 \mathrm{~cm}$ de canto mínimo. Al igual que cualquier tipo de losa prefabricada, el sistema diseñado puede apoyarse en muros de carga o en vigas de acero u hormigón.

En el caso del proyecto realizado presentado en este artículo la estructura se realiza con vigas de hormigón y pilares de acero. Las vigas de hormigón tienen como objetivo recibir las losas prefabricadas en su canto por los laterales, mientras que los pilares de acero permiten articular el encuentro con las vigas a fin de evitar agrietamientos y/o fisuras por posibles asentamientos que se pudieran producir por estar la vivienda cimentada sobre arcillas expansivas.

Todos los cálculos se realizaron teniendo en consideración la normativa EHE-o8 (16), así como el Código Técnico de la Edificación (17) (18).

\subsection{El proceso constructivo}

El encofrado cerámico: Las dimensiones métricas del encofrado responden a una anchura de 9 columnas de rasillas cerámicas (en color natural de $10 \times 20 \times 3 \mathrm{~cm}$ ), por una longitud variable; que se obtiene mediante la agregación de módulos de 4 filas de rasillas con un acero pre-ondulado de $2 \mathrm{~mm}$. Tras diversas muestras de prototipos se seleccionó aquella que se consideró más adecuada por su textura, luz y color.

Dimensiones: Las longitudes del tejido y de los moldes son prácticamente ilimitadas. En todo caso están condicionados por el sistema de transporte entre el taller de prefabricación y la obra. En este caso el programa de la vivienda determina las longitudes de las bóvedas: 3,79 m para el módulo de dormitorios, 5,04 m para el módulo de sala de estar y 1,29 m para el corredor de distribución de habitaciones. La anchura se limitó desde un comienzo a 1,00 m, debido a que responde a la mitad de la dimensión en ancho del contenedor de un camión de transporte de articulación simple.

Molde: Las primeras muestras realizadas con moldes curvados fueron desechadas debido a que el tejido cerámico sufría pequeños movimientos en la fase del vertido del hormigón; lo que provocaba distorsiones y filtraciones descontroladas del mortero de cemento, ocasionando coqueras entre las juntas de las piezas cerámicas (Figura 7). Por tal motivo se optó por diseñar un molde con forma poligonal (Figura 8), con la finalidad que las piezas del tejido de cerámica quedasen alojadas de manera totalmente coplanarias con la superficie del molde de acero. La longitud de los moldes alcanzaba los $8 \mathrm{~m}$ de longitud, y podían ser regulados mediante tabicas verticales ajustables a la dimensión requerida de cada revoltón.
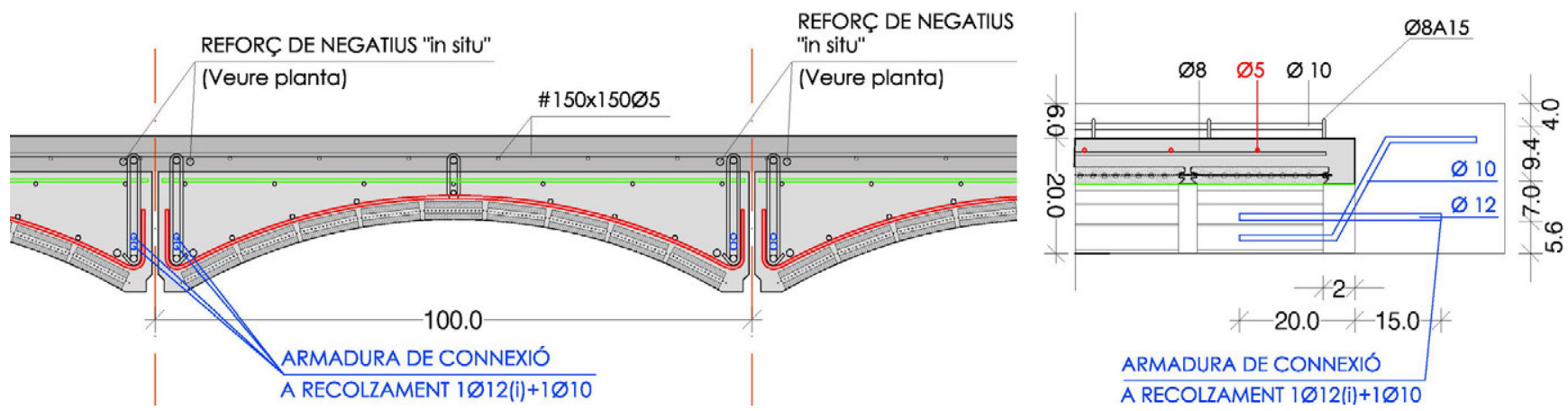

Figura 6. Esquema estructural del armado (Archivo TDB Arquitectura). 


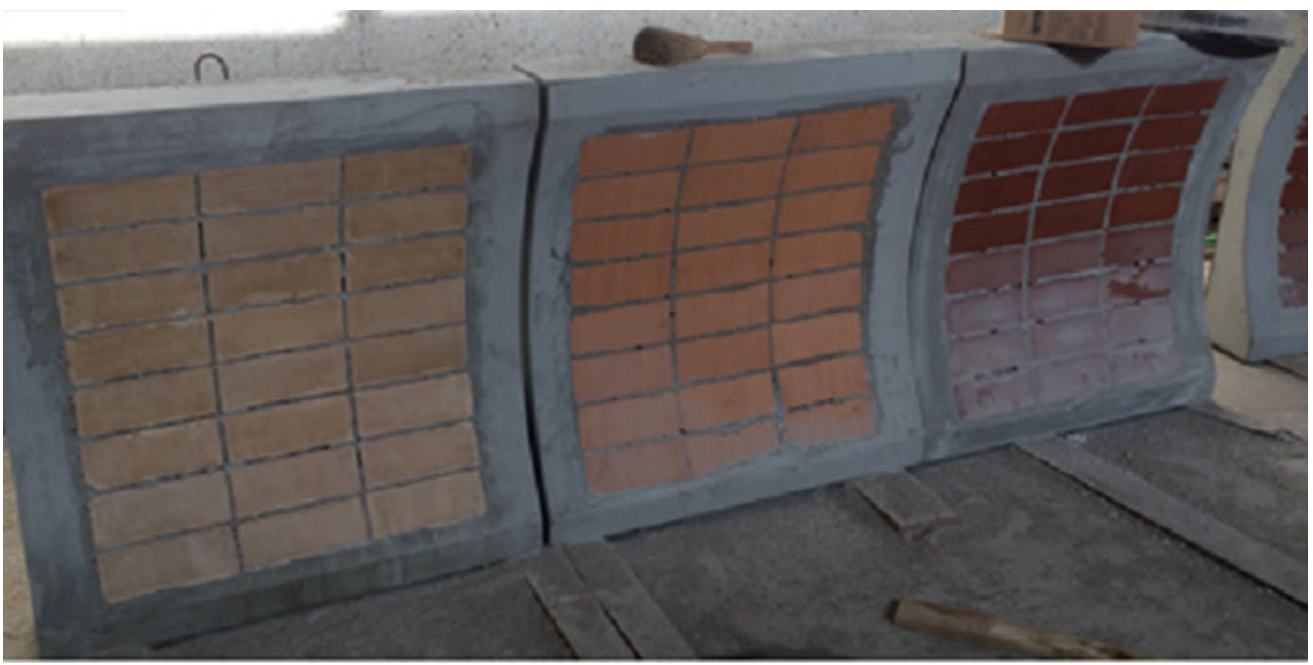

Figura 7. Coqueras en los primeros prototipos (fotografía del autor. Archivo TDB Arquitectura).

Alojamiento: La operación previa al alojamiento del tejido cerámico consiste en el tratamiento de la superficie de acero del molde mediante la aplicación de una veladura de aditivo retardador del fraguado del hormigón de carbonato o silicato de sodio (grupo III de la escala de retardadores de Forsen). Esta operación es absolutamente imprescindible para obtener juntas rehundidas de mortero respecto al plano de las rasillas cerámicas, produciéndose el mismo efecto visual que la tradicional llaga con espátula que realizaban los operarios tradicionales para resaltar la presencia de la cerámica (Figura 9, izquierda).

El alojamiento del tejido cerámico se realiza mediante puente grúa, mientras que los operarios «acompañan» el proceso de descenso hasta que la malla queda alojada sobre la superficie del molde (Figura 9, derecha).

Vertido: Existen dos aspectos determinantes en la operación del vertido: a) Árido del hormigón: no debe exceder de un diámetro de $5 \mathrm{~mm}$ debido a que el espacio intersticial entre las piezas de cerámica oscila entre los $15 \mathrm{~mm}$ y los $20 \mathrm{~mm}$ para evitar las coqueras mencionadas. Así, la dosificación utilizada es más propia de un mortero que de un hormigón, propiamente dicho. b) Sujeción del tejido cerámico: en la operación de vertido el tejido cerámico tiene tendencia a «flotar» sobre el mortero de cemento. Por este motivo se colocan una serie de barras y patillas de acero que inmovilizan por peso propio el tejido cerámico (Figura 9).

Desencofrado: Tras 24 horas de fraguado se retiran las bóvedas de los moldes mediante puente grúa (aprovechando las armaduras de espera preparadas para actuar como semi-losa del forjado). El retardante de fraguado que se había aplicado previamente sobre el molde facilita, tras el desencofrado, el lavado del intradós de la bóveda con agua a presión. Con ello, además de limpiar las superficies de las piezas de cerámica manchadas con el caldo del hormigón, se rebaja el mortero de cemento de las juntas de las bóvedas con el objetivo enfatizar la presencia de la cerámica. Se trata de una operación mecanizada que sustituye al tradicional «llagado» que realizaban los operarios manualmente (Figura 10, izquierda).

Transporte: Las losas abovedadas se acopian en vertical para ser transportadas en camiones. La capacidad de cada vehículo es de entre 21 y 24 elementos, lo que equivale a unos $125 \mathrm{~m}^{2}$ de forjado por trayecto. Una vez en obra, se colocan mediante elementos auxiliares de grúa móvil.

Colocación en obra: Las losas abovedadas son transportadas a obra con camiones de articulación simple. Mediante
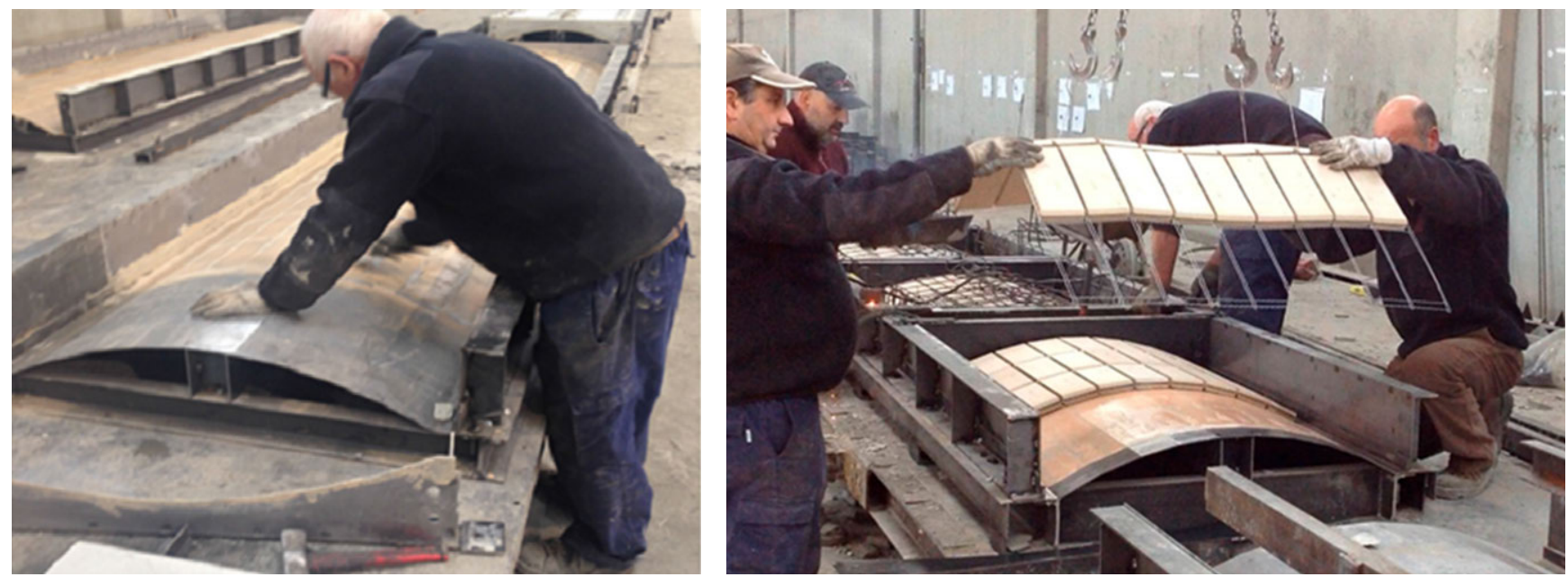

Figura 8. Molde de sección poligonal y aplicación de aditivo retardador (izquierda); alojamiento Flexbrick sobre el molde (derecha) (fotografía del autor. Archivo TDB Arquitectura). 

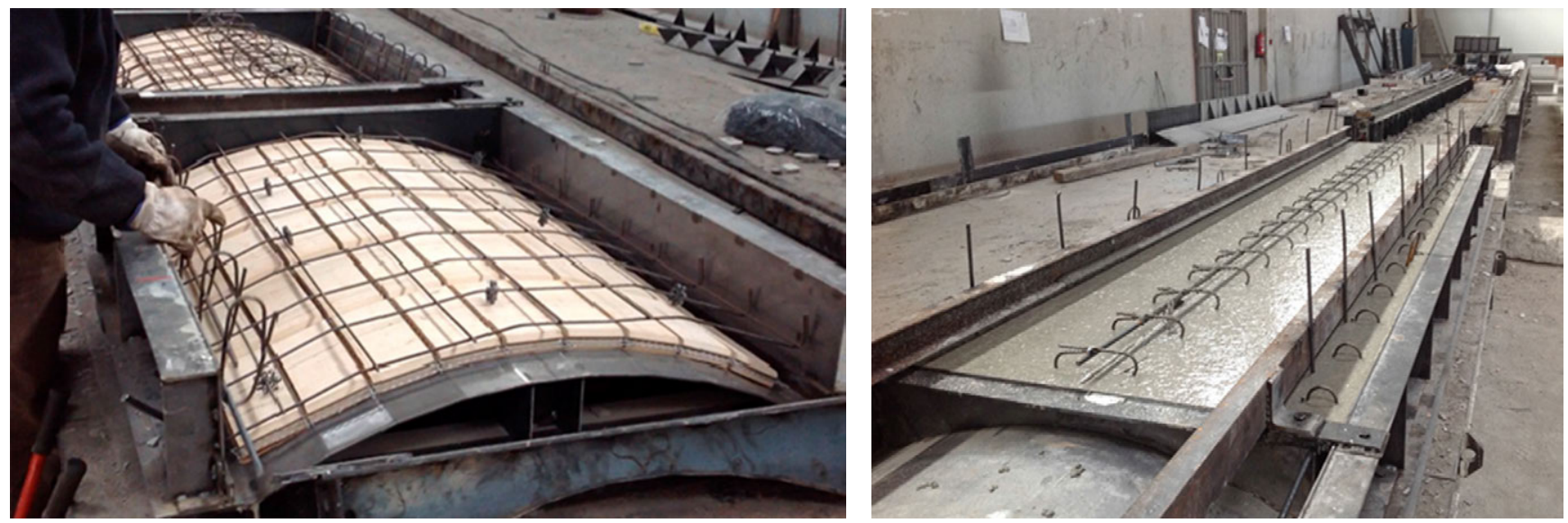

Figura 9. Colocación armadura estructural (izquierda); bóveda recién hormigonada (derecha) (fotografía del autor. Archivo TDB Arquitectura).
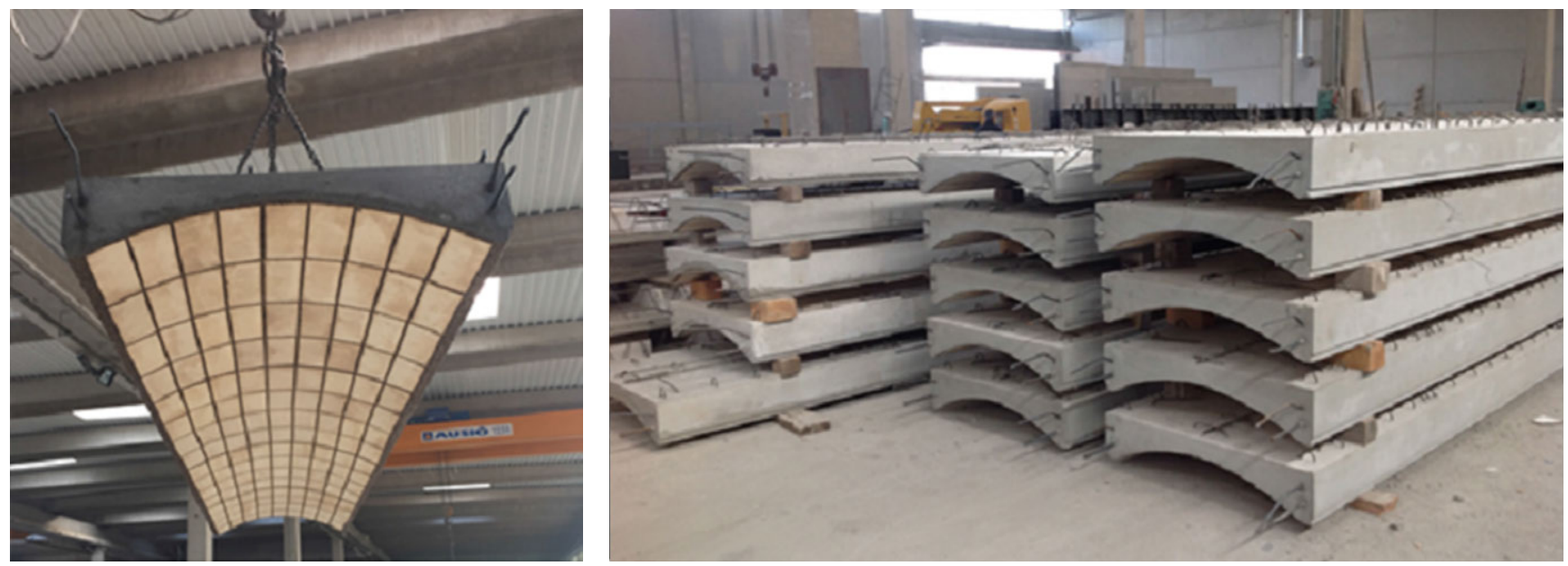

Figura 10. Desencofrado (izquierda); acopio de losas para transporte (derecha) (fotografía del autor. Archivo TDB Arquitectura).

grúa móvil pueden ser descargados del camión y en el mismo momento (sin requerir de acopio intermedio en obra), pueden alojarse sobre su posición definitiva. Una vez los elementos se han alojado sobre el encofrado puede procederse a la colocación del armado de los zunchos perimetrales, las armaduras de jácenas principales y el mallazo de reparto.

La experiencia llevada a cabo se ha realizado con jácenas encofradas y armadas in situ; lo que propicia la utilización del encofrado por mayor facilidad de ejecución. Cabe señalar que como evolución y mejora de este sistema se podría estudiar la ausencia del encofrado; lo que supondría una ventaja añadida al sistema desarrollado. Ello posiblemente sería factible si las jácenas principales se realizaran mediante elementos prefabricados.

El desencofrado in situ: En esta ocasión se utilizó encofrado de tarima de madera con la finalidad de facilitar los trabajos de replanteo de ejecución. Tras el hormigonado del forjado los tiempos de espera de fraguado son los mismos que para los forjados tradicionales realizados con losa unidireccional. El aspecto de la cara inferior del forjado tras el desencofrado es el que se muestra en la Figura 11, derecha.

Acabado: Para la obtención del acabado final de la bóveda han sido necesarias dos actuaciones complementarias; la primera es una limpieza superficial generalizada mediante cepi- llado manual para eliminar suciedades de las rasillas recibidas durante el transcurso de la obra. La segunda intervención complementaria ha consistido en el "entapetado" (disposición de tapetas de madera) de los bordes de los elementos para ofrecer un acabado más cálido (Figuras 13 y 14).

\section{LA EXPERIMENTACIÓN REAL. LA CASA CDS}

El contexto del encargo de la casa CDS (Catalan Dome System) se produce en un emplazamiento que corresponde a un entorno tipo resort en el ámbito del «PGA Catalunya Golf», en el término municipal de Caldes de Malavella. Se trata de una actuación en un contexto de modelo urbanístico propio de ciudad-jardín, ajeno al tejido urbano de ciudad tradicional mediterránea.

La tipología de vivienda más extendida del «PGA Resort» responde a la de vivienda unifamiliar aislada. Las edificaciones existentes del conjunto mantienen una clara línea arquitectónica contemporánea, en donde predomina la horizontalidad, el color blanco y la presencia de jardines privados.

Así, sobre la «deslocalizada arquitectura» extendida en el resort del PGA, en la casa CDS surge la actitud de adoptar el arraigado imaginario arquitectónico del revoltón cerámico, en una línea de experimentación que enlaza con las experiencias precedentes descritas en los párrafos anteriores. 

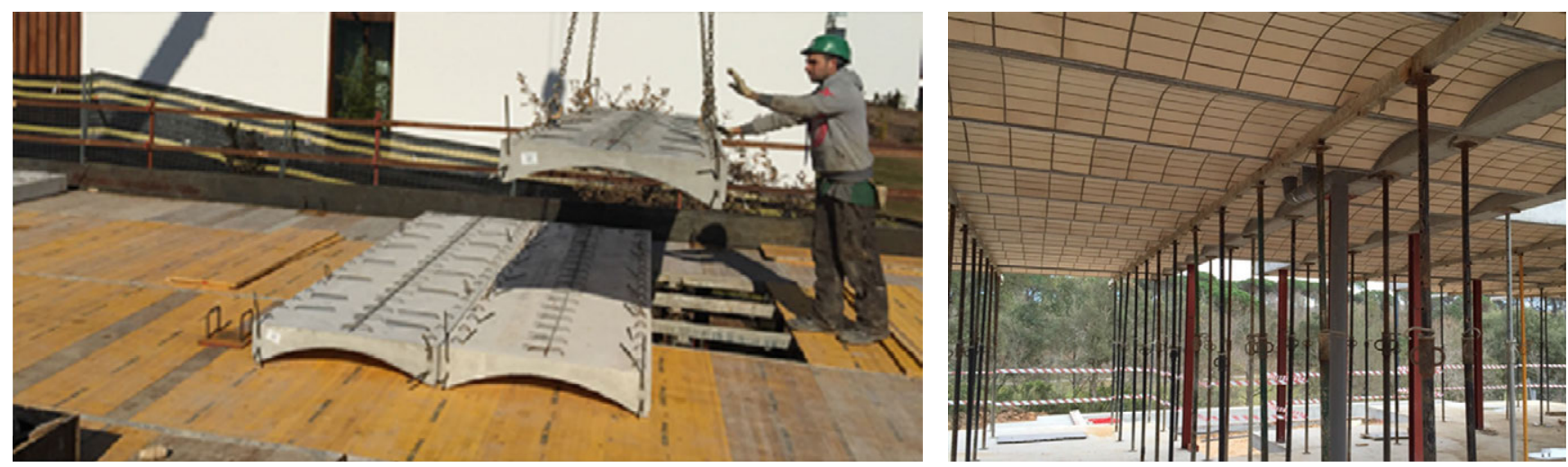

Figura 11. Colocación en obra (izquierda); aspecto final en obra (derecha) (fotografía del autor. Archivo TDB Arquitectura).

En la extensión del territorio catalán y, concretamente, en las comarques gironines próximas al emplazamiento del PGA, la utilización del «revoltón catalán» forma parte del «código genético» de la tradición constructiva. Su utilización se extendió tanto a los constructores más anónimos como a los arquitectos de la burguesía catalana de principios del siglo XX. La diversidad de propiedades constructivas del revoltón catalán son amplias y de interés vigente; entre ellos se pueden destacar dos aspectos que lo han universalizado: 1) la ejecución con sencillos medios auxiliares y 2) la capacidad para cubrir grandes luces.

El anhelo de explorar el espacio común entre la tradición y las nuevas tecnologías inspiró la obra que se presenta en este artículo, mostrando la inquietud de superponer la imagen poética de la tradición constructiva con las posibilidades que ofrecen las nuevas tecnologías.

La casa $C D S$ se proyecta desde la planta; desarrollando el diagrama paralelepípedo que ofrece el sistema de revoltón catalán para la construcción de forjados estructurales. Esta simple propiedad geométrica genera la métrica de la planta a dos niveles de escala arquitectónica: 1) «Módulos» básicos de estancias, y 2) agrupación de «Series» de diafragmas que admiten deslizamientos para adaptarse a los límites de las parcelas y a los desniveles del terreno (Figura 12).

\section{IDONEIDAD Y MEJORAS EN EL SISTEMA CONSTRUCTIVO}

El molde metálico (o rígido). La particularidad de este proceso es que el molde debe ser de sección poligonal idéntica al despiece del tejido metálico; lo que implica un molde diferente para cada solución de despiece y modulación de proyecto diferente. No obstante, ello no supone un inconveniente teniendo en cuenta que la repercusión del molde metálico sobre el coste del elemento no tiene una implicación crítica. El coste del molde para la prefabricación es de unos $100 € / \mathrm{m}^{2}$, pudiéndose utilizar repetidas ocasiones de manera prácticamente ilimitada (si se limpia bien después de la elaboración de cada elemento).

Tejido cerámico (con molde rígido) vs. moldes elásticos. Es una de las cuestiones relevantes a considerar, pues existe la alternativa de realizar este tipo de bóveda cerámica mediante moldes elásticos de caucho líquido (de la marca RECKLI o similar). Para este tipo de solución podría evitarse el molde metálico de sección poligonal, puesto que el molde elástico incorpora la cavidad para cada pieza de cerámica (que debe colocarse manualmente por los operarios). Este tipo de molde se obtiene a un coste de unos $400 € / \mathrm{m}^{2}$, y admite unas 40 puestas (calidad media). Contrariamente, como se ha descrito previamente, el molde metálico utilizado en la casa CDS ha tenido un coste de unos $100 € / \mathrm{m}^{2}$.
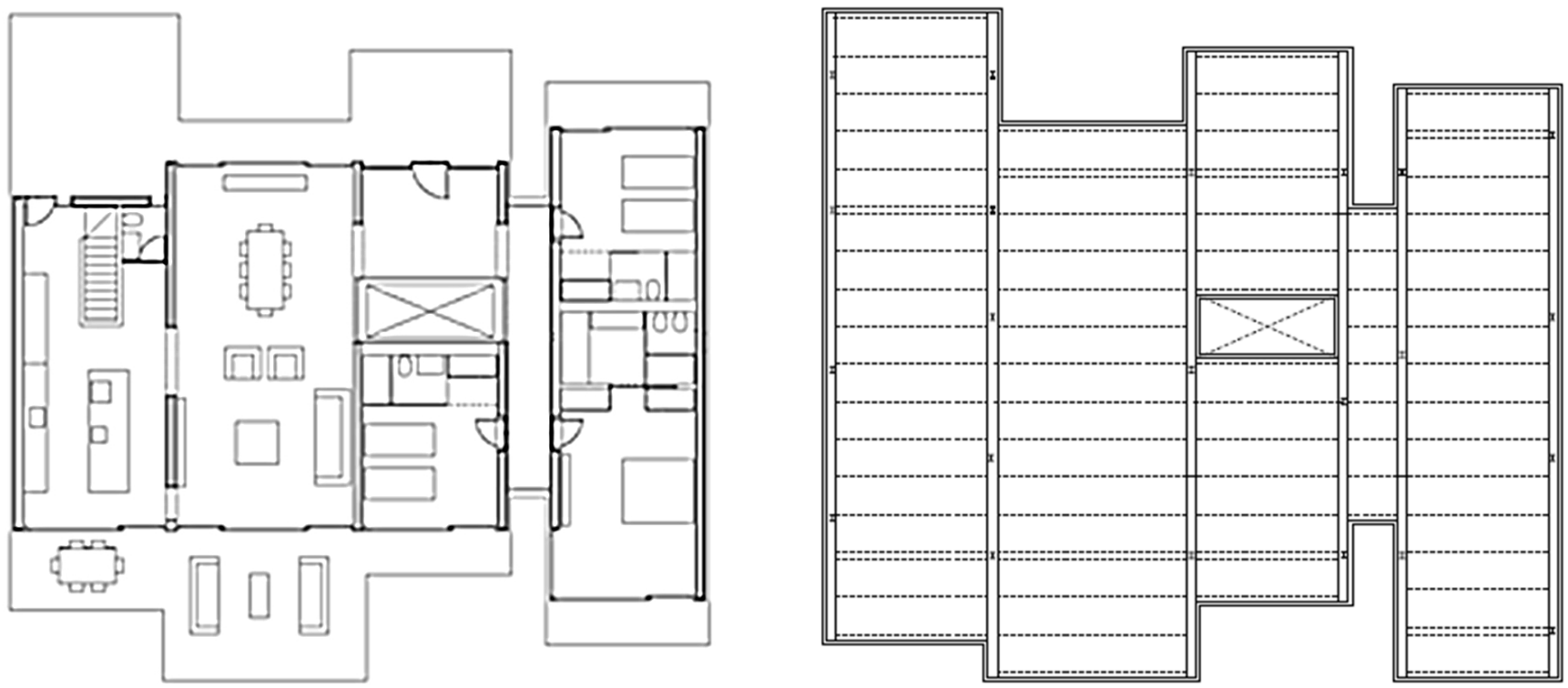

Figura 12. Planta de arquitectura (izquierda); esquema estructural (derecha) (Archivo TDB Arquitectura). 


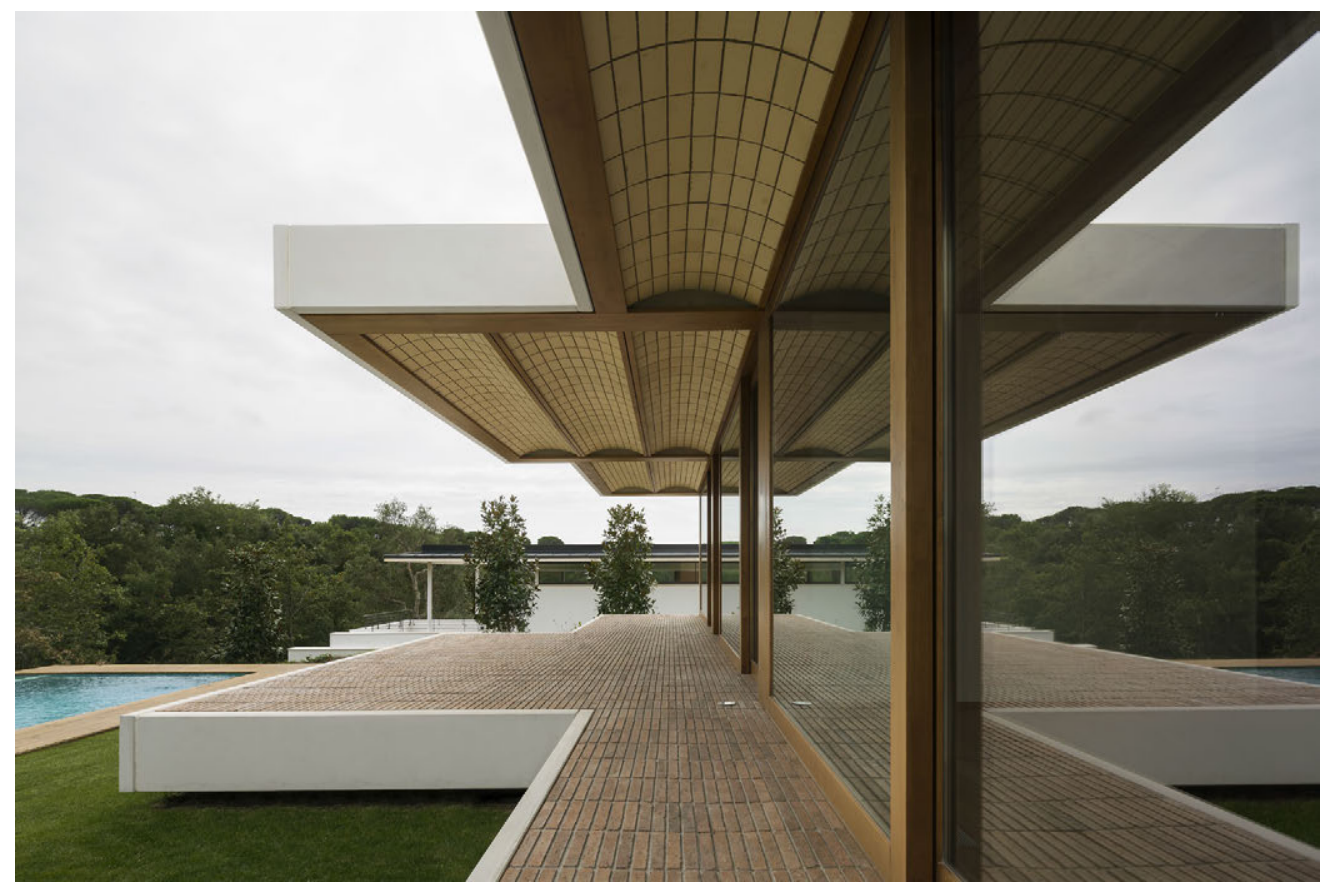

Figura 13. Aspecto final. Exterior (fotografía: Alejo Bagué. Archivo TDB Arquitectura).

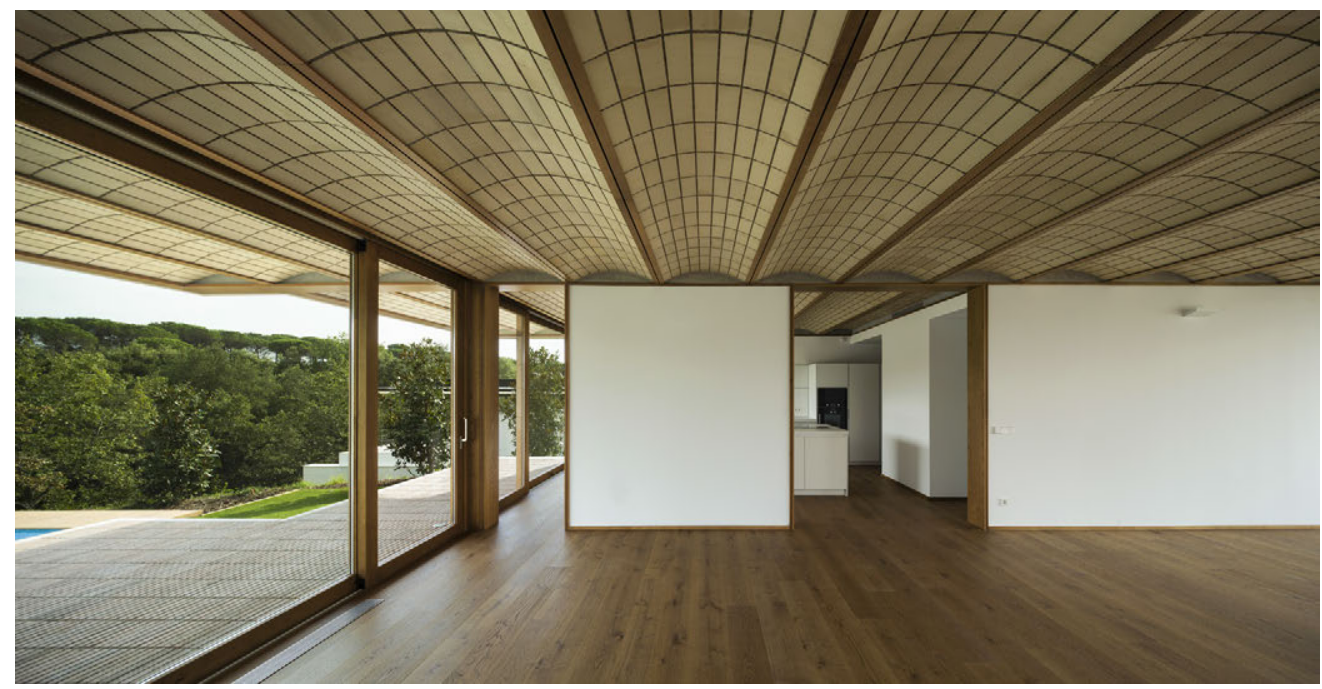

Figura 14. Aspecto final. Interior (fotografía: Alejo Bagué. Archivo TDB Arquitectura).

Según la experiencia de la empresa de prefabricación (Julián Arumí, S. L.), el coste de mano de obra, del material de hormigón y de las armaduras no difieren de manera significativa comparativamente entre ambos tipos de moldes. Por ello, para analizar la diferencia de coste entre uno y otro, nos podemos remitir a analizar únicamente el coste del propio molde y del material cerámico del acabado:

a) Moldes elásticos (caucho líquido): $400 € / \mathrm{m}^{2}$ (molde) $\times$ $30 \mathrm{~m}^{2 *} / 200 \mathrm{~m}^{2}$ (semi-losa) $+15 € / \mathrm{m}^{2}$ (pieza cerámica) $=75 € / \mathrm{m}^{2}$.

b) Moldes rígidos (metálicos): $100 € / \mathrm{m}^{2}$ (molde) $\times 30 \mathrm{~m}^{2 *} /$ $200 \mathrm{~m}^{2}$ (semi-losa $)+60 € / \mathrm{m}^{2}$ (tejido cerámico $)=75 € / \mathrm{m}^{2}$.

Este comparativo nos indica que el tipo de molde (elástico o rígido) no implica una gran diferencia de coste para una producción de $200 \mathrm{~m}^{2}$. Para producciones de cantidades significativamente mayores el molde elástico sería más competitivo, siempre dentro de la limitación de las 40 puestas mencionadas. No obstante, a partir de $1.500 \mathrm{~m}^{2}$ de producción (30 $\mathrm{m}^{2} \times 40$ puestas) el molde metálico tendería a ser más económico.

En cuanto a la expresividad tectónica del elemento, el tejido cerámico ofrece mayores posibilidades respecto al molde de caucho líquido, por cuanto al utilizarse una rasilla plana (y no piezas volumétricas para garantizar la adhesión al hormigón) la variedad de acabados se presenta mucho más amplia. Esto es, mientras que en el caso del molde elástico la posibilidad de variedad geométrica reside en el mismo molde, en el sistema de molde metálico (o rígido) la variedad se obtiene por las múltiples posibilidades que permite el tejido cerámico.

\footnotetext{
* Son necesarios un mínimo de $30 \mathrm{~m}^{2}$ de molde para un proceso de prefabricación eficiente para una producción de $200 \mathrm{~m}^{2}$ (casa CDS).
} 
La posibilidad de «tecnificación». Esta acepción se refiere en este caso a la capacidad de incorporar tecnología en el elemento de prefabricado de hormigón. Las líneas de producción en cadena del taller sugieren la posibilidad (previamente a la fase de hormigonado) de la incorporación de elementos complementarios tales como fijaciones, articulaciones, instalaciones, conducciones, etc. Lo que induce a imaginar que en el banco de trabajo del proceso de prefabricación del taller, además del montaje y hormigonado, pueden incorporarse otros trabajos que habitualmente se realizan en la obra.

\section{REFLEXIONES COMPLEMENTARIAS}

$\mathrm{Al}$ margen de las consideraciones técnicas, tras la experiencia de prefabricación con bóveda de tejido cerámico, surge la reflexión sobre la idoneidad del proceso en términos socioeconómicos.

Una vez finalizado el proceso de construcción, surge el debate acerca de la eficacia del proceso y sobre la posibilidad de sucesivas experiencias con tejido cerámico.

La conclusión de los responsables de la empresa encargada de la prefabricación consiste en que es necesario perseverar y profundizar en este tipo de procesos con tejido cerámico. El motivo que ampara esta afirmación se encuentra en la dicotomía Ordenamiento vs. Oficio.

$\mathrm{Si}$ «Oficio» es aquella facultad que requiere de un proceso de aprendizaje sobre la ejecución del trabajo a realizar; «Ordenamiento» sería la organización de un proceso de ejecución, cuya aplicación no requiere de un aprendizaje prolongado ni especializado; pues en éstos la especialización subyacería en la sistematización del mismo proceso y no en las habilidades por parte de quienes lo deben llevar a cabo.

Así, el «Oficio» puede implicar una dificultad de los procesos industriales y constructivos en la medida en que existe la ten- dencia a la desaparición de largos períodos de aprendizaje, así como de permanencias prolongadas de mismos operarios en la misma industria (o en los procesos de ejecución).

Contrariamente, el «Ordenamiento» se fundamenta en que los procesos industriales o constructivos soportan su propia especialización; de manera que quien deba acometerla se sirve del protocolo establecido para su realización, y no tanto en su propia capacidad de aprendizaje o habilidades.

Por tanto, Ordenamiento vs. Oficio, en realidad, confronta las nociones de Tecnología vs. Técnica (en un sentido arquitectónico-constructivo); sin subestimar que la técnica puede estar en la base para desarrollar procesos tecnológicos inspirados en el imaginario de la construcción tradicional.

Al mismo tiempo existe un aspecto adicional que se viene sedimentando inexorablemente desde finales del siglo XIX en el ámbito de la construcción y de la arquitectura; son los referidos al campo de la fenomenología (19). Esto es, aquellas referencias de nuestras raíces culturales que participan y determinan la praxis de nuestras actividades cognoscitivas y artísticas. Es el motivo por el cual, por ejemplo, nos sentimos atraídos por las antigüedades, por la arquitectura vernácula o por los materiales tradicionales. Así, la evolución de nuestros edificios avanzan entre los paradigmas de la tradición y del progreso, respondiendo a patrones que configuran nuestro imaginario arquitectónico.

Tradición y progreso, pues, constituyen un binomio sobre el que evaluamos la idoneidad de los sistemas constructivos. Mientras que la tradición es sinónimo de durabilidad, de raíces culturales o de eficacia energética y constructiva, el progreso sugiere procesos industrializados, preocupación medioambiental o implementación de las tecnologías en nuestro hábitat cotidiano. Ambas contienen aspectos deseables, por lo que somos receptores del legado de integrar y hacer compatible las mejores propiedades de la tradición constructiva con el avance y evolución de las nuevas tecnologías.

\section{REFERENCIAS}

(1) Bassegoda, M. (1946). La bóveda catalana. Zaragoza: Institución Fernando el Católico.

(2) Luis Sert, J., Torres Clavé, J., Subirana, J. (1960). El dispensario antituberculoso de la calle Torres Amat (1934-38). Cuadernos de Arquitectura (40). Clínicas y dispensarios, http://www.raco.cat/index.php/CuadernosArquitectura/article/ view/109500.

(3) Bassegoda i Nonell, J. (2001). Rafael Guastavino Expósito (1873-1950). En Huerta Fernández, S. (ed.): Las bóvedas de Guastavino en América, pp. 17-18. Madrid: Instituto Juan Herrera.

(4) Huerta Fernández, S. (2006). La construcción tabicada y la teoría cohesiva de Rafael Guastavino. En Huerta Fernández, S. (ed.): Escritos sobre la construcción cohesiva y su función en la arquitectura, pp. 15-62. Madrid: Instituto Juan Herrera.

(5) Pizarro, M., Rueda, O. (2013). Una nueva expresividad de las bóvedas tabicadas. Las Escuelas Nacionales de Arte de la Habana. Arquitectura y Urbanismo, 34 (1).

(6) García, M., Beltrán, F. (2014). La prefabricación de bóvedas de ladrillo. Una utopía latinoamericana. Rita_ (2): 92-99.

(7) Prozorovich, F. (2011). La conservación del patrimonio histórico moderno. Entre la regla y la excepción. Vitrubius. Arquitextos. ISSN: $1809-6298$.

(8) García, J., González, M., Losada, J.C. (2012). Arquitectura y construcción tabicada en torno a Eduardo Sacristie. Informes de la Construcción, 64 (525): 35-50.

(9) Sacriste, E., Kechichian, P., Mackintosh, G. (1977). Viviendas con bóveda. Buenos Aires.

(10) González Ortiz, H. (2003). Arquitectura en precario. La propuesta de Carlos González Lobo. Ciencia Ergo Sum 11 (1): 117-124. ISSN: 1405-0269.

(11) Cordero, T. (2014). Arquitectura de un futuro pasado. Estudio comparativo entre diferentes sistemas constructivos de bóvedas y láminas. Valencia: Escuela Técnica Superior de Arquitectura de Valencia. 
(12) www.cyted.org/.

(13) Flexbrick: http://www.flexbrick.net/en/.

(14) Sarrablo, V., Colom, J. (2011). Los tejidos cerámicos y la casa Mingo. Detail (6): 640-645.

(15) Sarrablo, V. (2011). Casa Mingo. ConArquitectura (40): 45-52.

(16) EHEo8 (2008). Instrucción del Hormigón Estructural. Ministerio de Fomento.

(17) CTE-DB (2009). Código Técnico de la Edificación. Documento Básico. Ministerio de Fomento.

(18) CTE-DB-AE (2009). Código Técnico de la Edificación. Acciones en la edificación. Documento Básico. Ministerio de Fomento.

(19) Ábalos, I. (2001). La buena vida. Visita guiada a las casas de la modernidad. Barcelona: Gustavo Gili, S. A. 